\title{
Prevalence of Abnormal Cervical Cytology among Postnatal Clinic Attendees at the University of Calabar Teaching Hospital, Nigeria
}

\author{
Boniface Uji Ago ${ }^{1 *}$, Aniekan Etokidem², Godwin Ebughe ${ }^{3}$ \\ ${ }^{1}$ Department of Obstetrics and Gynaecology, University of Calabar Teaching Hospital, Calabar, Nigeria \\ ${ }^{2}$ Department of Community Medicine, University of Calabar, Calabar, Nigeria \\ ${ }^{3}$ Department of Pathology, University of Calabar Teaching Hospital, Calabar, Nigeria \\ Email: ^bonifaceago@yahoo.com, etokidem@etokidem.com, gobeng02@gmail.com
}

How to cite this paper: Ago, B.U., Etokidem, A. and Ebughe, G. (2016) Prevalence of Abnormal Cervical Cytology among Postnatal Clinic Attendees at the University of Calabar Teaching Hospital, Nigeria. Open Access Library Journal, 3: e2992. http://dx.doi.org/10.4236/oalib.1102992

Received: August 19, 2016

Accepted: September 2, 2016

Published: September 5, 2016

Copyright $\odot 2016$ by authors and Open Access Library Inc.

This work is licensed under the Creative Commons Attribution International License (CC BY 4.0).

http://creativecommons.org/licenses/by/4.0/ (c) (i) Open Access

\section{Abstract}

Background: Carcinoma of the uterine cervix is a deadly disease, but it is preventable. Cervical cytology especially at postnatal visit can lead to its early detection and prompt treatment. Methods: This was a prospective cross sectional study of postnatal women to determine the prevalence of abnormal cervical cytology and their socio-demographic characteristics. Patients were recruited from the antenatal clinic and counseled on the need for postnatal cervical smear. Only those who consented and met the inclusion criteria were followed up in the postnatal clinic. Institutional ethical clearance was also obtained. Researcher administered questionnaire was used to collect the socio-demographic features while Aye's spatula and endocervical brush were used to collect specimen from the ectocervix and endocervix respectively and smeared on individually labeled glass slides. The samples were treated using the conventional Papanicolaou staining method and reported using the Bethesda system. Researcher-administered questionnaire generated data as well as results of cytology were analyzed using Epi-Info version 3.5.1.0 (CDC Atlanta USA 2008). Colposcopy was done for smear positive patients. Results: There were 100 women screened. Three percent had low grade squamous intraepithelial lesion. There were no abnormal findings from the Colposcopy of the three patients. Ninety-five percent of the women had no prior knowledge of cervical cancer and Pap smear. Two percent of the women had a family history of cervical cancer and both patients died of the disease due to lack of facilities for treatment. Conclusions: Cervical cancer is a preventable disease but women of the sub-region are under-screened. Postnatal Pap smear is a necessary tool in its early diagnosis and treatment.

\section{Subject Areas}

Gynecology \& Obstetrics 


\section{Keywords}

Cervical Cancer, Prevention, Pap Smear, Postpartum Women, UCTH

\section{Introduction}

Invasive squamous cell carcinoma of the cervix is the end stage of a process that begins with atypical transformation of cervical epithelium at the squamocolumnar junction, progressing to cervical intraepithelial neoplasia (CIN) of advancing grades and then the invasive disease [1].

Cervical cancer is the third most common cancer in women throughout the world and it is the leading cause of cancer death among women in underdeveloped countries [2] [3].

In Nigeria, cervical cancer accounts for $70 \%$ of female genital cancers with an annual incidence of 25,000 new cases or 480 cases per week [4].

In a previous study in this hospital (UCTH), cancer of the uterine cervix was responsible for $2.76 \%$ of gynaecological admissions with $82.5 \%$ of the patients presenting with late disease where little or nothing could be done for them [2].

Cervical cancer exerts health, economic and emotional burden on the sufferer and her family [5].

The higher incidence of cervical cancer in developing countries is largely due to the lack of effective screening programmes to detect pre-malignant lesions, and treat them before they progress to invasive cancer. Nigeria does not have a national (or even any regional) screening programme [6].

There is convincing evidence that cytologic screening programs are effective in reducing mortality from carcinoma of the cervix [6]. The extent of the reduction in mortality achieved is related directly to the proportion of the population that has been screened [7]. In fact, all studies worldwide show that screening for cervical cancer decreases mortality and incidence of the disease [6]-[9].

Screening decreases cervical cancer mortality by detecting preinvasive disease [6] [7] at the beginning of a screening program in a previously unscreened population. It detects prevalent cases of invasive cancer in earlier stages [8]. Although, it has not been proved in a prospective randomized study, all investigators credit screening as a major contributor to this reduction in death rate [7].

In contrast to the industrialized world, cancer of the cervix remains the primary cancer killer in women in third-world countries [10].

The paucity of effective screening programs is the bane of the late presentation of cervical cancer in the underdeveloped countries. In Nigeria, only about $5 \%$ of the women have ever been screened [5].

A successful screening program must be directed at a disease with a screening test [11]. A suitable disease must be one that has serious consequences, as most cancers do. Treatment must be available so that when such therapy is applied to screen-detected 
(preclinical) disease, it will be more effective than when applied after symptoms of the disease have appeared. Also, the preclinical phase of the disease must be long enough that the chances are good that a person will be screened. Cervical cancer is a suitable disease for screening [6]-[11].

There must also be a suitable screening test as defined by simplicity, acceptability to patients, low cost, and high validity [1].

Despite the effectiveness of cervical cytology screening programs, there are several limitations of Papanicolaou smear screening. A single Pap smear has a sensitivity of only about $51 \%$ but its specificity is about $98 \%$ [7] [12]. The false-negative rate of a Pap smear is about $20 \%$, but this is mostly from sampling error [13]. It is noted that even with this limited sensitivity, if three consecutive tests are negative, there is less than a $1 \%$ chance that the patient will have a cervical abnormality [14].

Some of the sampling errors are due to timing, method of collection, transportation and laboratory handling and interpretation of results [7].

The cytologic sample should not be collected during the menstrual period. The patient should avoid vaginal medications, vaginal contraceptive or douches during the 48 hours before the appointment and intercourse is not recommended on the night before or the day of the examination [10]. From the standpoint of obtaining ideal cytology, postpartum Pap smears should not be performed until at least 6 or even 8 weeks after delivery, by which time the cervix has undergone reparative changes [7].

One of the drawbacks of Pap smear is the high false negative rate [7]. This associated with the cost of mass screening, the delay in retrieving results and paucity of trained cyto-technicians had been cited as the reason for alternative screening methods such as VIA (Visual Inspection with Acetic acid (either with or without magnification), automated pap, cervicography and Human Papilloma Virus (HPV) DNA testing [7]. Since its approval by the Food and Drug Administration in the United States of America in 1996, liquid-based cytology (ThinPrep) replaced conventional cytology as the standard for cervical cancer screening. Reasons cited for this change include its superior specimen quality and sensitivity for detection of low-grade squamous intraepithelial lesions (LSIL), high-grade squamous intraepithelial lesions (HSIL), and other lesions [15].

Liquid-based cytology was developed as an alternative method, and although numerous studies have compared the accuracy of liquid based cytology and conventional cytology, results have been inconsistent [16]. A large randomized study has found that, compared with the conventional Papanicolaou (Pap) test, liquid-based cytology is not more sensitive or specific in detecting precancerous lesions or cervical cancer [16]. In this large population-based cluster randomized control trial, the screening performance of Pap tests and liquid based cytology in terms of test positivity rates, histological detection rates, and positive predictive values (PPVs) were compared. The advantage that liquid-based cytology has over conventional Pap smear includes reduced unsatisfactory rates, the possibility of concomitant testing for HPV or other molecular markers, and the possibility for computer-assisted screening [16]. 
Increased recognition of human papillomavirus (HPV) as the primary cause of cervical cancer has led to internationally endorsed incorporation of HPV-DNA testing, in addition to the Pap test, into new screening and triage guidelines. In the United States, it is FDA approved as an adjunct to cervical cytology screening in women over the age of 30, and for the triage of women with Pap smears identified as atypical squamous cellsundetermined significance (ASC-US). The American College of Obstetricians and Gynecologists (ACOG) Practice Bulletin (April 2005) noted that women who have a negative Pap and HPV have a 1:1000 chance of having unidentified cervical intraepithelial neoplasia (CIN) 2 or 3 [15]. The 2001 Bethesda system [17] reports abnormal squamous cytology as either atypical squamous cells (ASC, subdivided into 2 categories: ASCUS and ASC-H), squamous intraepithelial lesions (SIL, subdivided into 2 categories: LSIL [CIN1] and HSIL [CIN2, 3]), and squamous cell cancer (SCC).

The relevance of doing Pap smear in pregnancy has been well researched. In a study of 253 women referred for colposcopy during pregnancy for ASC-US/LSIL. Colposcopic impression revealed normal status or LSIL in 93\% of the patients; consequently, cervical biopsy revealed HSIL in 6 patients. Postpartum Pap smears were normal in $32 \%$ of patients, unchanged in $65 \%$, and HSIL in $3 \%$. Postpartum biopsy confirmed that none of the patients had invasive cancer, while 4 had high-grade dysplasia [18]. This study indicates that ASC-US/LSIL does not require colposcopic evaluation during pregnancy.

Another study, conducted at Athens University, reported on 208 patients with abnormal cytology during pregnancy who were evaluated over 10 years. It showed that $37 \%$ of patients had CIN2,3. After conservative management with colposcopy and cytology during pregnancy and postpartum, $38 \%$ of the cases persisted, and $62 \%$ of the cases regressed to CIN2. No invasive cancers developed during follow-up [19].

Another study assessed the management and evolution of CIN during pregnancy and postpartum in patients with antenatal abnormal Pap smear who were evaluated with colposcopy, laser vaporization, or loop electrosurgical excision procedure (LEEP). It reported that $36 \%$ of the patients with CIN1 and $48 \%$ of the patients with CIN2 regressed postpartum. There was one case of microinvasion diagnosed postpartum, corresponding to an antepartum diagnosis of CIN3 [20].

Although Counselling for postpartum Pap smear is a component of routine postpartum assessment and care, [21] most women in our setting do not come for cervical smear cytology [6]. Some of the reasons cited include, ignorance and lack of awareness about cancer, and the screening methods as well as cultural and religious inhibitions [6]. Of note in Nigeria is that, health workers that have been trained as cytoscreeners are very few, with less than 70 anatomic pathologists to cover all autopsy, histology and cytology services [6], little wonder then that results are often delayed causing undue frustration and loss to follow-up.

There is no doubt that prevention is better than cure. Intensified cervical screening especially the postpartum smear is a veritable method of preventing the sequelae and carnage caused by cervical cancer. 
Symptoms of invasive cervical cancer include vaginal bleeding, vaginal discharge, postcoital bleeding, and pelvic pain, but approximately $20 \%$ of patients are asymptomatic. It is easy to disregard abnormal bleeding in pregnancy and attribute it to a pregnancy-related cause, such as threatened abortion and antepartum haemorrhage, so delayed diagnosis is common [22] [23].

Cone biopsy is problematic in pregnancy because of increased risks for haemorrhage, abortion and preterm labour [22] [23].

In the early stage disease, uterus conserving fertility preserving surgeries are available. [6] In stage 1a1 disease, Loop Electrosurgical Excision Procedure (LEEP) or cone biopsy with adequate disease free margin, is adequate. In stage 1a2 disease, LEEP must be accompanied by laparoscopic lymph node dissection [6].

Vaginal radical trachelectomy (VRT) with laparoscopic pelvic lymphadenectomy was introduced for the first time in 1986, it is associated with low morbidity, with a tumour recurrence of $5 \%$ and a mortality rate of $3 \%$ [6]. Pregnancies of up to $70 \%$ have been reported [6]. These cases are only possible where patients are adequately screened and disease detected at an early stage. In a literature review that included cases diagnosed up to 12 months postpartum, an incidence of invasive cancer of 4.54 cases per 10,000 pregnancies was reported [24]. One in 34 cases of cervical cancer was diagnosed during or within 12 months of pregnancy [22].

Visual inspection with Acetic acid (VIA) is an alternative to Pap smear. Its accuracy is comparable and results are immediately available. VIA is a promising alternative to Pap smear in developing countries because it is inexpensive and fast and requires a low level of training and no special equipment [25]. It requires only acetic acid, a speculum, and a light source-does not require microscope, laboratory, specially trained technicians, pathologists, transport, reporting, and supplies. However, false positives may overload the referral system and result in unnecessary treatments.

The limitations of the conventional Pap smear can be reduced by proper method of collection, fixing, transport, laboratory handling, staining and interpretation of results.

Postpartum cervical cytology may be the only opportunity for women of reproductive age to be screened and benefit from early intervention.

The incidence of abnormal cytology in pregnancy varies from 1.2 to 1.9 percent. However, the prevalence in Ile-Ife was 2.6 percent [26]. Screening for cervical cancer remains the only way for early diagnosis, and prompt and effective treatment.

This study sought to find out the prevalence of abnormal cervical cytology among postpartum women attending post-natal and maternal and child health clinics in UCTH after due counselling.

The consideration here is that, most women present late with cervical cancer in our center. A screening program in the reproductive age especially after childbirth may be the only opportunity for screening these women. With proper method of collection, fixing, transport, laboratory handling, staining and interpretation, the benefits of conventional smear may be optimized. 


\section{Objectives of the Study}

The overall objective was to offer opportunistic screening, diagnose early stage cervical cancer and provide early and appropriate intervention.

The specific objectives are:

1) To determine the prevalence of abnormal cervical smears among postnatal clinic attendees in UCTH.

2) To determine the socio-demographic characteristics of the women who had postpartum cervical smear.

\section{Methodology}

\subsection{Study Design}

This was a prospective cross-sectional study aimed at determining the prevalence of abnormal cervical cytology among postnatal and maternal and child health clinic attendees in the University of Calabar Teaching Hospital.

\subsection{Study Area}

This study was conducted at the Maternity annex of the University of Calabar Teaching hospital which is located at the centre of Calabar metropolis. Calabar is the capital of Cross River State. It is popularly called 'the people's paradise, Canaan city or the tourism destination'. Its people are hospitable, and engage in all aspects of human endeavour. The University of Calabar Teaching Hospital (UCTH) provides tertiary health care for about 1.8 million people in Cross River State and serves as a referral centre for adjourning states of Akwa Ibom and Benue as well as the Republic of Cameroun. It also serves as a referral centre for private clinics, primary and secondary health centres within the catchment areas.

Although there is a cancer registry in Calabar, only few women go for screening. Screening opportunities are provided by the Department of Obstetrics and Gynaecology, UCTH, and the Medical Women Association through their out-reach programs.

This study area is representative of the geographical and socio-demographic characteristics of the reproductive age women in Calabar.

\subsection{Sampling Technique}

Women were recruited at the antenatal clinics during pregnancy and at the postnatal wards after delivery and followed up at postnatal clinic, and maternal and child health clinics. They were counselled for the test. The inclusion criteria are: all postpartum patients of all parities; the exclusion criteria are: those who are on follow-up following a prior positive cervical smear, and those who object.

Patients were selected by systematic sampling of every other woman who met the inclusion criteria between August 2012 and October 2012. Each of the women was given a Questionnaire that was interviewer administered after which an informed consent form was signed. 


\subsection{Sample Collection}

With the procedure well explained to the patient, the chaperone/nurse placed the patient in the dorsal position with her legs flexed to expose the vulva. Under aseptic procedure, gloves were worn by this researcher and a sterile disposable speculum passed to expose the cervix. The vagina and cervix are inspected.

Scrapings of the cervix were collected using Ayre's spatula and endocervical brush. The scrapings were spread on a clean glass slide and immediately fixed in $95 \%$ alcohol to prevent drying.

All samples so collected and fixed were properly labeled and taken in the alcohol medium to the pathology laboratory for cytological processing staining, microscopy and analysis, with the assistance of a cytopathologist.

Materials for processing cervical smear: Ethanol, hydrochloric acid, aluminium hydroxide, xylene, cover lips, microscope slides, methanol, and microscope. The procedure of conventional Pap smear was followed.

\subsection{Determination of Sample Size}

The minimum sample size was determined using the formula for single proportion

$$
\mathrm{n}=\mathrm{z}^{2} \mathrm{pq}
$$

$\mathrm{d}^{2}$

Where $\mathrm{n}=$ minimum sample size;

$\mathrm{z}=$ standard normal deviate, which at the $95 \%$ confidence level is 1.96 ;

$\mathrm{p}=$ the proportion of respondents with the desired characteristics. In this case the existing prevalence is $2.6 \%(0.026)$.

$\mathrm{Q}=1-\mathrm{p} \times(0.974)$.

$\mathrm{d}=$ margin of error allowed which is set at $0.05 \%$ for this study

$=1.962 \times 0.026 \times 0.974 \times(0.05)^{2}$

$=38.9$.

Making allowance for attrition rate of $15 \%$, this became 44.73 .

For the purpose of this research, 100 patients were studied.

\section{Data Analysis}

After the preparation of the slides, the findings were reported using the Bethesda classification. The information extracted from the questionnaire such as age, marital status, parity, educational status, menstrual and obstetric history as well as gynaecologic history was computed using Epi_Info version 3.5.1.0 for analysis using frequency tables. The mean age of patients was also measured.

\subsection{Ethical Consideration}

Since this study involves human subjects, ethical committee approval of the University of Calabar Teaching Hospital was obtained.

Confidentiality was strictly adhered to. Patients were adequately counseled about 
cervical cancer and the objectives of this study were explained to them. Those with abnormal smears were treated accordingly.

\subsection{Study Limitation}

Proper specimen collection and fixation of cells is essential for interpretation. This study was done with the assistance of a cytopathologist. One of the study constraints was the cost of Pap smear. An unforeseen challenge was the refusal of some women to be screened after counseling even when the cost was borne by the researchers.

\section{Results}

The results are presented in two parts. First the socio-demographic characteristics of the women studied and then the cytological report of the study.

Table 1(a) shows the demographic variables studied. Sixty one percent of the women were aged 20 - 34 years (this is shown in Table 1(b)). The mean age of the women was 31.29 years with a variance of 38.511 and a standard deviation of 6.2057. The modal age group was 36 years old.

Forty six per cent were multiparous (para 2 - 4), while $29 \%$ were grandmultiparous.

Fifty one per cent of the women had their menarche at age 13 , while $22 \%$ had theirs at age 15 and above.

Most of the women (51\%) had tertiary level of education.

Eight-eight percent of the women studied were married.

Only two patients (2\%) had a positive family history, and whereas 13 patients (13\%) have heard of cervical cancer, only five (5\%) had knowledge of cervical cancer.

None of the women studied had a previous pap smear.

Eight respondents (8\%) had abnormal vaginal discharge post-partum. They all had endocervical swab taken for microscopy, culture and sensitivity and treated accordingly.

\section{Cytological Report}

They were one hundred (100) patients studied.

The results were reported using the Bethesda 2001 system.

The specimen type was the conventional smear (Pap smear).

Each specimen was evaluated for adequacy, that is, whether it was satisfactory or unsatisfactory for evaluation.

The general categorization was to identify cellular patterns which might depict infective organisms like bacteria (Actinomyces spp.), Fungi (Candida spp.) or viruses (herpes simplex, human Papilloma virus); reactive cellular changes associated with inflammation; and epithelial cell abnormality.

In this study, there were three types of result presentations:

1) Negative for intraepithelial lesion,

2) Inflammatory,

3) Epithelial cell abnormality identified as low grade intraepithelial lesion. 
Table 1. Demographic variables of patients who had postpartum cervical smear.

(a)

\begin{tabular}{|c|c|c|c|}
\hline Variable & Frequency & Percent (\%) & Mean age \pm SD (range) \\
\hline \multicolumn{4}{|c|}{ Age range in years } \\
\hline $20-24$ & 19 & 19 & $31.3 \pm 6.2$ \\
\hline $25-29$ & 20 & 20 & $(20-42)$ \\
\hline $30-34$ & 22 & 22 & \\
\hline$\geq 35$ & 39 & 39 & \\
\hline Total & 100 & $100 \%$ & \\
\hline \multicolumn{4}{|l|}{ parity } \\
\hline 1 & 25 & 25 & \\
\hline $2-4$ & 46 & 46 & \\
\hline$\geq 5$ & 29 & 29 & \\
\hline Total & 100 & $100 \%$ & \\
\hline \multicolumn{4}{|c|}{ Menarche (in years) } \\
\hline$\leq 11$ & 7 & 7 & \\
\hline 12 & 8 & 8 & \\
\hline 13 & 51 & 51 & \\
\hline 14 & 12 & 12 & \\
\hline$\geq 15$ & 22 & 22 & \\
\hline Total & 100 & $100 \%$ & \\
\hline \multicolumn{4}{|c|}{ Educational status } \\
\hline Primary & 12 & 12 & \\
\hline secondary & 37 & 37 & \\
\hline Tertiary & 51 & 51 & \\
\hline Total & 100 & $100 \%$ & \\
\hline \multicolumn{4}{|c|}{ Marital status } \\
\hline Married & 88 & 88 & \\
\hline Single & 12 & 12 & \\
\hline Total & 100 & $100 \%$ & \\
\hline \multicolumn{4}{|c|}{ Family history of cervical cancer } \\
\hline Yes & 2 & 2 & \\
\hline No & 98 & 98 & \\
\hline Total & 100 & $100 \%$ & \\
\hline \multicolumn{4}{|c|}{ Heard of cervical cancer } \\
\hline Yes & 13 & 13 & \\
\hline No & 87 & 87 & \\
\hline Total & 100 & $100 \%$ & \\
\hline
\end{tabular}




\section{Continued}

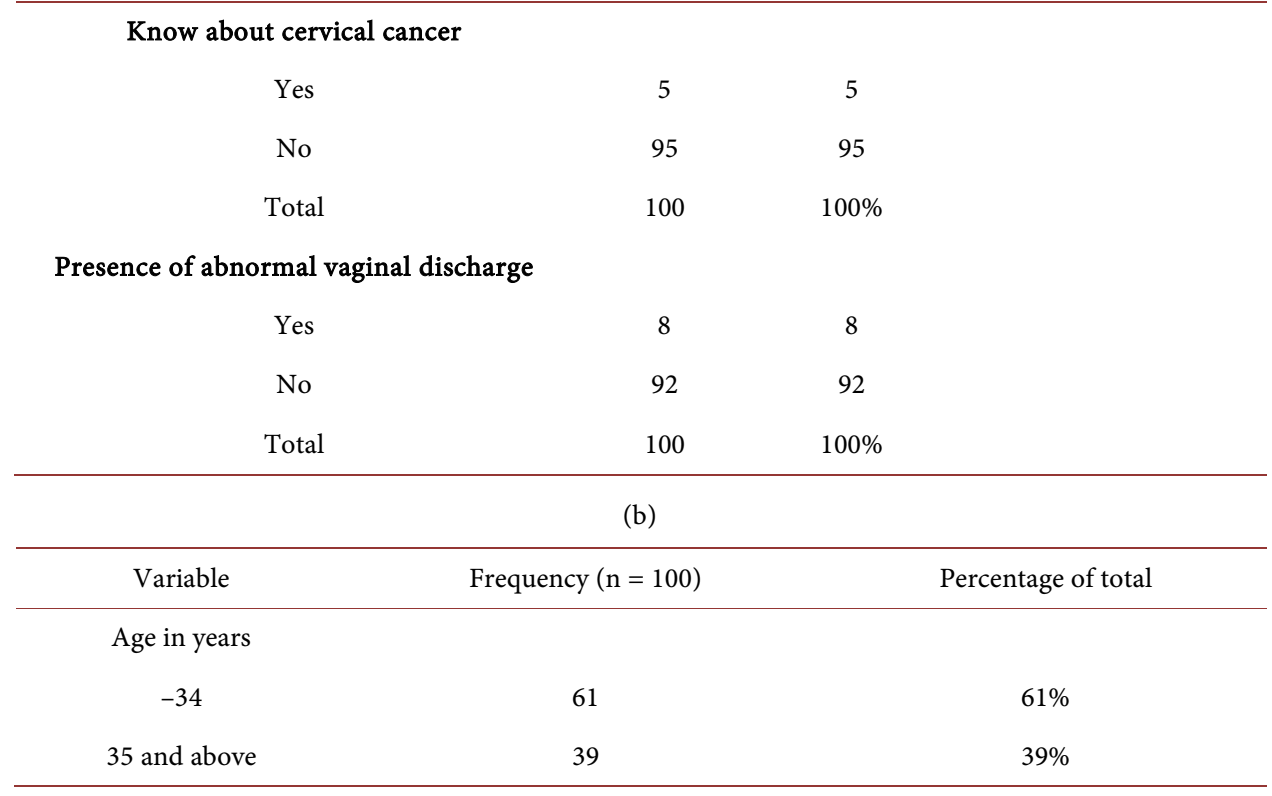

Table 2. Report of the cytological evaluation.

\begin{tabular}{cccccc}
\hline variable & number & total & $\%$ total & Chi-square & p-value \\
\hline negative & 97 & 100 & 97 & 65.320 & 0.000 \\
abnormal & 3 & 100 & 3 & & \\
\hline
\end{tabular}

Table 3. Demographic characteristics of the patients with abnormal smear.

\begin{tabular}{cccccccc}
\hline patient & age & menarche & parity & Marital status & Edu status & Abn v disch & Family history \\
\hline A & 30 & 11 & 2 & Married & tertiary & + & + \\
B & 35 & 12 & 4 & Married & Tertiary & + & + \\
C & 42 & 15 & 5 & married & secondary & + & + \\
\hline
\end{tabular}

In the final analysis, both $\mathrm{A}$ and $\mathrm{B}$ were grouped as NEGATIVE while $\mathrm{C}$ was ABNORMAL.

Of the one hundred (100) samples studied, only three (3) were abnormal. This is shown in Table 2.

The prevalence of abnormal cytology among this group of postnatal attendees was three percent $(3 \%)$.

The Demographic characteristics of the three postnatal women identified as Patient A, B and C with abnormal Pap smear (low grade squamous intraepithelial lesion) showed that they were aged 30, 35 and 42 respectively. This is shown in Table 3.

All three patients were married. Their sexual behaviour was not significantly different from the other women studied.

All three women had abnormal vaginal discharge contributing three of eight (37.5\%) patients with abnormal vaginal discharge in this study. 
The relationship between those affected and the number of their sexual partners could not be determined in this study. However, two of the affected women constituted two of two (100\%) of women with positive family history of cervical cancer.

\section{Discussion}

The prevalence of abnormal cervical cytology in this study was 3\%. This is close to the prevalence of $2.6 \%$ reported in 1985 [26].

Cancer of the uterine cervix is a global problem with significant morbidity and mortality if it is not detected before it reaches an advanced stage.

Cervical cancer not only exerts a huge economic, psychosocial and emotional burden on the affected women but more specifically compromises their reproductive aspirations. When cervical cancer occurs in a young woman desirous of pregnancy, effective care is often a huge challenge. Where the disease is early, prognosis is excellent for stage 1a1 because nodal metastasis is under $0.5 \%$ [27]. The incidence of lymph node metastasis in stage $1 \mathrm{a} 2$ disease is $7 \%$ with an overall recurrence rate of $5 \%$ [28]. Following therapy, the 5 -year survival of cervical cancer for stage 1 disease is between $86.5 \%$ and $98.7 \%, 64.7 \%-68.8 \%$ for stage II, $40.4 \%$ - $43.3 \%$ for stage III, and $15 \%-19.5 \%$ for stage IV.

Most of our patients with cervical cancer are poor and under-privileged, unable to afford investigations not to talk of radiotherapy sessions and are easily lost to follow-up [2]. Where patients are able to afford radiotherapy services, this facility is either absent, in a state of disrepair or over-subscribed with long "awaiting" list. Some of these patients either die before the course or die of radiotherapy side effects [2].

The mean age of the patients in this study was 31.29 years, and the modal age group was 36 years. This is close to the mean age of patients with pre-invasive cancer of the cervix, which is 34 years [29].

The three women who had low grade squamous intraepithelial lesion were aged 30 , 35 and 42 with parities $2^{+3}, 4^{+2}$, and $5^{+3}$ respectively.

Cervical intraepithelial lesion is usually asymptomatic and they are rarely seen on routine examination. Since the transit time from CIN to invasive cancer is unpredictable on an individual basis and the morphology of the lesion does not predict which lesions will progress or regress, follow up is crucial. It has been reported that the transit time from CIN1 to CIN3 is six months to 3 years, and that $57 \%$ of CIN1lesions regress, $32 \%$ persist and $11 \%$ progress.

The three patients in this study who had low grade cervical squamous intraepithelial lesion underwent colposcopy. The colposcopy findings were normal and the patients were to have a repeat cervical cytology in three months.

Awareness on cervical cancer is poor. Although $51 \%$ of the women screened had tertiary education, surprisingly, only 13 out of the 100 women have heard of cervical cancer before. Of this thirteen, only five had a fair knowledge of cervical cancer. There is great need therefore for an accelerated effort to improve public awareness on cervical cancer, and to make it a part of routine gynaecological examination procedure in all 
women aged thirty and above, unless otherwise unnecessary. $88 \%$ of the women were married but none of all the women studied had done a previous Pap smear.

HPV DNA typing was not done on them, because the hospital did not have facility for this testing at the time, so it was difficult explaining the cause of the abnormal smear. However, the patients are on follow up. One of the limitations of this study was cost. However, an unforeseen challenge was the reluctance of women to voluntarily accept screening even when it was free. Most of them wanted to come back at a later date after making up their minds, but never did. There was that fear for the unknown.

Sensitive public health campaigns would be required to convince parents to allow their teenage daughters to be vaccinated against a sexually transmitted infection such as Human Papillomavirus. Some pro-abstinence and religious groups may be opposed to the vaccination of young girls, fearing that it may promote promiscuity [30].

An HPV prophylactic vaccine is unlikely to benefit women who have already been exposed to the relevant virus type. It may therefore take a generation before all women at risk of cervical cancer can be vaccinated and even then a proportion will remain unvaccinated. It is not clear to what extent older women with prior HPV exposure could be protected by vaccination. Cervical screening will, therefore, continue to play an important role in the fight against cervical cancer. HPV prophylactic vaccination may not be $100 \%$ effective and will probably not protect against all HPV types. There will, therefore, be a need for continued cervical screening in a vaccination era. Future screening strategies may depend upon primary HPV testing with cytology being reserved for those women who are HPV positive [30].

It has been reported that one HPV shot may be enough to protect against cervical cancer [31].

The various screening methods such as Pap smear, Visual inspection of the cervix with either Acetic acid (VIA), VIAM or Lugol's iodine (VILI), Liquid-based cytology, and HPV testing combined with diagnostic tests such as colposcopy, cervicography, Papnet and cervical biopsy histology offer secondary prevention opportunities.

If screening becomes a routine postnatal examination procedure and the cost of screening built into a formal postnatal check-up bill, it will remove this unwillingness and uncertainty of patients about screening. This unwillingness is reminiscent of the pre-PEPFAR days of HIV screening, where the response to voluntary counselling and testing was poor. But the provision of free antiretroviral drugs and elaborate campaigns on counselling and behaviour modification have improved uptake. For screening to be meaningful there must be available cheap options of treatment.

In Nigeria, today the campaigns for free cervical cancer screening are sporadic, and generated by different interest groups. However after the screening, the affected women are left to seek treatment by themselves.

There is also a paucity of gynaecologic oncology experts to effectively handle this increasing population of affected women. Most centres in Nigeria do not have cryotherapy, loop electrosurgical excision procedure (LEEP), and radiotherapy services, among many other challenges. Palliative care is perhaps the only form of care for patients with 
advanced disease. This service may either be non-existent, rudimentary or not properly composed in some centers in Nigeria.

\section{Conclusions}

Cervical cancer is a burden especially in our subregion, but it is largely preventable by doing regular cervical cytology to identify the preinvasive lesion at which stage prompt intervention is lifesaving.

Effective screening programmes are the mainstay of the war against cervical cancer.

Vaccination against Human Papilloma Virus (HPV), and HPV-DNA typing are encouraging but still expensive.

Training of personnel and provision of facilities for gynecologic oncology in each of the tertiary hospitals are necessary to stop the carnage caused by this disease in Nigeria. The burden of cervical cancer is heavy, but it is surmountable.

\section{References}

[1] Berek, J.S. and Hacker, N.F. (2000) Epidemiology and Biostatistics of Cervical Cancer. Practical Gynaecologic Oncology, 3rd Edition, Lippincott Williams and Wilkins, 7, 258.

[2] Ago, B.U., et al. (2013) Cancer of the Uterine Cervix at the University of Calabar Teaching Hospital, Calabar, Nigeria. Cancer Research Journal, 1, 37-40.

[3] Cohn de Herzog, T.J. (2001) New Innovations in Cervical Cancer Screening. Clinical Obstetrics and Gynecology, 44, 538-549.

[4] Adewole, I.F. (2003) Epidemiology, Clinical Features and Management of Cervical Cancer. In: Okonofua, F. and Odunsi, K., Eds., Contemporary Obstetrics and Gynaecology for Developing Countries, Women's Health and Action Research Center, Benin, 289-315.

[5] Omigbodun, A.O. (2009) Epidemiology of Cervical Cancer. Colposcopy Training Workshop, Calabar.

[6] Omigbodun, A.O. (2010) Guidelines for Cervical Cancer Screening. How to Take a Good Pap Smear. Update Course WACS Abuja.

[7] Hasanzadeh, M. and Behtash, N. (2005) Cervical Cancer Screening. Cancer Therapy, 3, 231236.

[8] Parkin, D.M., Nguyen-Dinh, X. and Day, N.E. (1985) The Impact of Screening on the Incidence of Cervical Cancer in England and Wales. British Journal of Obstetrics and Gynaecology, 92, 150-157.

[9] Cecelia, H. Boardman. Cervical Cancer. Medscape Medical. http://www.medscape.com/article/253513

[10] Disaia, P.J. and Creasman, W.T. (2002) Preinvasive Disease of the Cervix. In: Disaia, P.J., Creasman, W.T., Eds., Clin Gynaecol Oncology, 6th Edition, St Louis Mosby Year Book, 1, 1-35.

[11] Cole, P. and Morrison, A. (1980) Basic Issues in Population Screening for Cancer. Journal of the National Cancer Institute, 64, 1263-1272.

[12] Apgar, B.S., Spitzer, M., et al. (2002) Colposcopy: Principles and Practice. An Integrated Textbook and Atlas. Saunders Company, Philadelphia, 52-56.

[13] Garcia, A.A. Cervicalcancer. http://emedicine.medscape.com/article/1253513

[14] Rock, J.A. and Jones III, H.W. (2003) Cervical Cancer Precursors and Their Management. 
TeLinde’s Operative Gynaecology, 9th Edition, Lippincort Williams \& Wilkins, 45, 13511373.

[15] Monk, B.J. Cervical Cancer Screening. HPV Resource.org

[16] Siebers, A.G., et al. (2009) Pap Tests and Liquid-Based Cytology in Detecting Precancerous Lesions. JAMA, 302, 1757-1764. http://dx.doi.org/10.1001/jama.2009.1569

[17] Apgar, B.S., Zoschnick, L. and Wright, T.C. (2003) The 2001 Bethesda System Terminology. American Family Physician, 68, 1992-1999

[18] Jain, A.G., Higgins, R.V. and Boyle, M.J. (1997) Management of Low-Grade Squamous Intraepithelial Lesions during Pregnancy. American Journal of Obstetrics \& Gynecology, 177, 298-302. http://dx.doi.org/10.1016/S0002-9378(97)70190-X

[19] Vlahos, G., Rodolakis, A., Diakomalis, E., et al. (2002) Conservative Management of Cervical Intraepithelial Neoplasia $\left(\mathrm{CIN}_{2-3}\right)$ in Pregnant Women. Gynecologic and Obstetric Investigation, 54, 78-81. http://dx.doi.org/10.1159/000067715

[20] Paraskevaidis, E., Koliopoulos, G., Kalantaridou, S., et al. (2002) Management and Evolution of Cervical Intraepithelial Neoplasia during Pregnancy and Postpartum. European Journal of Obstetrics and Gynecology and Reproductive Biology, 104, 67-69. http://dx.doi.org/10.1016/S0301-2115(02)00058-1

[21] Postpartum Care (2006) The Royal Women's Clinical Practice Guideline. The Royal Women's Hospital 2006. www.thewomens.org.au

[22] Berek, J. and Hacker, N.F. (2000) Berek Practical Gynaecologic Oncology. Lippincott Williams and Wilkins Publishers, Philadelphia.

[23] Kwame-Aryee, R. (2005) Carcinoma of the Cervix. In: Kwawukume, E.Y. and Emuveyan, E.E., Eds., Comprehensive Gynaecology in the Tropics, Graphic Packaging, Ghana, 412428.

[24] Hacker, N.F., Berek, J.S., Lagasse, J.D., Charles, E.H., Savage, E.W. and Moore, J.G. (1982) Carcinoma of the Cervix Associated with Pregnancy. Obstetrics and Gynaecology, 59, 735746.

[25] Jeronimo, J., Morales, O., Horna, J., et al. (2005) Visual Inspection with Acetic Acid for Cervical Cancer Screening Outside of Low Resource Settings. Revista Panamericana de Salud Pública, 17, 1-5. http://dx.doi.org/10.1590/s1020-49892005000100001

[26] Olatunbosun, O.A., Ayangade, S.O. and Okwerekwu, G.A. (1985) Cytologic Screening for Cervical Neoplasia in Pregnancy. Tropical Journal of Obstetrics and Gynaecology, 5, 63-66.

[27] EL-Gbobasky, A. and Herrington, S. (2005) Cervical Cancer: Epidemiology and Molecular Characteristics. In: Studd, J., Ed., Progress in Obstetrics and Gynaecology, Elsevier Science Ltd., Amsterdam, Vol. 16, 323-341.

[28] Buckley, S.L., Tritz, D.M., et al. (1996) Lymph Node Metastases and Prognosis in Patients with Stage $\mathrm{IA}_{2}$ Cervical Cancer. Gynecologic Oncology, 63, 4-9. http://dx.doi.org/10.1006/gyno.1996.0268

[29] Azodi, M. and Ray, W. (2003) Premalignant Lesions of the Lower Genital Tract. In: Okonofua, F. and Odunsi, K., Eds., Contemporary Obstetrics and Gynaecology for Developing Countries, Women's Health and Action Research Centre, Benin, 253-288.

[30] Vaccination against Cervical Cancer. Scientific Advisory Committee Opinion Paper 9. RCOG Guidelines. https://www.rcog.org.uk/en/guidelines-research-services/guidelines/sip9/

[31] Brooks, M. (2013) One HPV Shot Maybe Enough to Protect against Cervical Cancer. Medscape Medical News Online. http://www.medscape.com/viewarticle/813780 
Submit or recommend next manuscript to OALib Journal and we will provide best service for you:

- Publication frequency: Monthly

- 9 subject areas of science, technology and medicine

- Fair and rigorous peer-review system

- Fast publication process

- Article promotion in various social networking sites (LinkedIn, Facebook, Twitter, etc.)

- Maximum dissemination of your research work

Submit Your Paper Online: Click Here to Submit

Contact Us: service@oalib.com 